CrossMark

\&lick for updates

Cite this: Food Funct., 2016, 7, 3111

\title{
Reduction of anxiety-like and depression-like behaviors in rats after one month of drinking Aronia melanocarpa berry juice
}

\author{
Mirko Tomić, ${ }^{a}$ Đurđica Ignjatović, ${ }^{a}$ Gordana Tovilović-Kovačević, ${ }^{a}$ \\ Dijana Krstić-Milošević, ${ }^{a}$ Slavica Ranković, ${ }^{\text {b Tamara Popovićc }}$ and Marija Glibetićb
}

\begin{abstract}
The treatment of mood and anxiety disorders by nutraceuticals is gaining growing awareness. Berries of Aronia melanocarpa (Black chokeberry) and their extracts, exceptionally abundant in diverse phenolic compounds, have become famous for the highest in vitro antioxidant activity among fruits and notable health benefits (e.g. anti-diabetic, anti-inflammatory, cardioprotective). This study was designed to investigate the behavioral effects of month-long unlimited consumption of Aronia master juice (AJ) and/or juice reconstruct without polyphenols $(R J)$, in young male rats. AJ was initially evaluated for its content of phenolic compounds by spectrophotometric assays and HPLC-DAD. Rats that were supplied with three various water concentrations of $A J$ and RJ, respectively: $20 \%+0 \%$ (ARO group), $5 \%+15 \%$ (RAJ) and $0+$ $20 \%$ (PLC), were compared with those which consumed only water (CTL). Daily drinking of AJ solution was significantly elevated from the second or third week onward, which was most expressed in the ARO group. Only this group displayed behavioral variations, manifested by certain hyperactivity in open field tests and prominent reductions of anxiety-like behaviors in the elevated plus maze. The ARO rats also expressed an alleviation of depression-like behavior in forced swimming tests. These findings demonstrate the beneficial behavioral effects of the one-month-long free drinking of phenolic-rich AJ in rats $(>20 \mathrm{ml}$ per kg b. mass daily) that may be recognized as stimulating, anxiolytic-like and antidepressant-like. The in vitro assays suggested that $M A O-A / M A O-B$ inhibitions by the phenolic compounds of $A J$ might be the possible in vivo mechanisms for such behavioral actions.
\end{abstract}

\begin{abstract}
Received 8th March 2016 Accepted 27th May 2016

DOI: $10.1039 / \mathrm{c} 6 \mathrm{fo} 00321 \mathrm{~d}$

www.rsc.org/foodfunction
\end{abstract}

\section{Introduction}

Medical literature suggests that mental illnesses have become almost epidemic in the developed world. ${ }^{1}$ Based on clinical documentation, it was estimated that nearly $20 \%$ of both the adult and youth population in the USA had some form of mental ailment in $2013 .^{2}$ Mood and anxiety disorders are two kinds of psychiatric diseases that attract most of the attention of the public and medical community, which is based on the evidence that more than half of mankind may be affected by either of these complaints during their life. ${ }^{1}$ Besides psychotherapy, the first-line option in the management of these illnesses is pharmacotherapy, where a growing number of psychoactive drugs exerts a substantial degree of beneficial

\footnotetext{
${ }^{a}$ Department of Biochemistry, Institute for Biological Research "S.Stankovic", University of Belgrade, Bul. Despota Stefana 142, 11000 Belgrade, Serbia. E-mail: mitomic@ibiss.bg.ac.rs; Fax: +381 11 2078433; Tel: +381 112078306 ${ }^{b}$ Centre of Excellence in Nutrition and Metabolism, Institute for Medical Research, University of Belgrade, Tadeuša Košćuška 2, 11000 Belgrade, Serbia
}

effects. $^{3,4}$ Unfortunately, most of these drugs are commonly associated with unwanted side-effects and many individuals exhibit refractory or intolerant responses. ${ }^{1,3,4}$ Therefore, a certain part of research on psychiatric diseases has focused on the investigation of alternative therapies or adjunctive strategies, which could improve clinical outcomes. ${ }^{5}$ The use of nutraceuticals is one of the main approaches to that effect. ${ }^{5-8}$

The Aronia shrub, a member of the Rosaceae family, has become a very popular cultivar in Eastern and Northern Europe, where it is grown as an ornamental plant and industrial source of natural coloring agents. ${ }^{9}$ The berry of Aronia melanocarpa Elliott (Black chokeberry; the genus name Aronia has been replacing this common name) is rich source of polyphenols, especially anthocyanins, which are correlated with high antioxidant activity. ${ }^{9,10}$ Aronia has a simple anthocyanin spectrum, consisting almost exclusively of cyanidin glycosides such as cyanidin-3-arabinoside and cyanidin-3-galactoside, but has a manifold higher anthocyanin content in comparison with other berry fruits. ${ }^{10,11}$ Proanthocyanidins and hydroxycinnamic acids (chlorogenic and neochlorogenic acids) are also 
found in greater amounts while flavonols (quercetin glycosides) and flavan-3-ols (epicatechin) are usually present as minor components in Aronia berries. ${ }^{10,12}$ A number of experimental and clinical studies revealed that anthocyanins themselves, such as anthocyanin-rich Aronia extracts, exhibited diverse potential health benefits, including cardioprotective, anti-diabetic and anti-inflammatory effects..$^{9,13-15}$ Some limited studies have also shown the effects of Aronia juices on the behavior and cognitive functions of experimental animals. ${ }^{16,17}$ Our observation that certain other polyphenolrich fruit extracts, although lower in phenolics when compared to Aronia, have provoked a number of animal and human studies that investigated their behavioral/psychological effects, ${ }^{18-21}$ inspired us to explore those particular influences of the juice of Aronia.

This behavioral study was carried out on young male rats, which were provided for one month with unlimited consumption of water dilutions $(20 \%, v / v)$ of one of the following: master Aronia juice (AJ) with a high concentration of phenolic compounds, juice reconstruct without phenolic compounds $(\mathrm{RJ})$, or their combination $(\mathrm{AJ}: \mathrm{RJ}=1: 3)$, where the control group of animals was supplied with pure water. The influences of the drinking variants on the overall activity and locomotion, as well as on anxiety-like and depression-like behaviors, were evaluated using appropriate animal behavioral models and are discussed later. AJ was originally assayed for total phenolic constituents and proanthocyanidins, while the particular phenolic compounds were identified and quantified by the HPLC$\mathrm{DAD}$ method. The capacity of $\mathrm{AJ}$ to inhibit in vitro monoamine-oxidase enzymes, MAO-A and MAO-B, was estimated and compared with analogous effects of the several phenolic compounds found in AJ. The relations between the potential in vivo MAO inhibition by the phenolic compounds of Aronia and the experimental behavioral parameters registered in this study are discussed.

\section{Materials and methods}

\subsection{Materials}

A commercial product ("Nutrika", Belgrade, Serbia), obtained by pressing of fresh Aronia berries and presented as a pure and concentrated Aronia master juice (AJ), was used to prepare drinking solutions in our lab. The manufacturer's analytical data for the AJ batch used in this study, regarding the nutritional values of the most abundant components of $\mathrm{AJ}$, were used to prepare RJ. It contained the exact same amounts of particular sugars, organic acids, minerals and vitamins as AJ, but without the bioactive phenolics (in $\mathrm{g} \mathrm{L}^{-1}$ ): glucose 45.0, fructose 28.0, sorbitol 50.0, citric acid 1.21, K-citrate-monohydrate 6.0; in $\mathrm{mg} \mathrm{L}^{-1}$ : vitamin $\mathrm{C} 29.2$, vitamin $\mathrm{B} 10.38$, vitamin B2 0.71 , vitamin B6 0.44, niacin $0.39, \mathrm{CaCl}_{2} \times 2 \mathrm{H}_{2} \mathrm{O} 559, \mathrm{MgCl}_{2}$ $550, \mathrm{NaCl} 760$. Both master beverages, AJ and RJ, were stored at $4{ }^{\circ} \mathrm{C}$ during the study. All chemical reagents applied were of analytical grade and produced either by "Sigma" (USA) or "Serva" (Germany).

\subsection{Analysis of phenolic compounds in $\mathbf{A J}$}

The total phenolics in AJ were determined by a modified FolinCiocalteu method and presented in $\mathrm{mg}^{-1}$ of gallic acid equivalents. ${ }^{22,23}$ Identification and quantification of individual phenolic compounds were performed by chromatographic analysis (Agilent series 1100 HPLC instrument with a DAD detector) on a reverse phase Zorbax SB-C18 (Agilent) analytical column $(250 \mathrm{~mm} \times 4.6 \mathrm{~mm}$ i.d., $5 \mu \mathrm{m}$ particle size $){ }^{23,24}$ Before its injection into the HPLC system, AJ was filtered through a $0.45 \mu \mathrm{m}$ membrane filter. The analysis of flavonoids and hydroxycinnamic acids was performed with a mobile phase that consisted of solvent A (1\%, v/v solution of orthophosphoric acid in water) and solvent $\mathrm{B}$ (acetonitrile) using an elution gradient according to the previously published method: 90-80\% A 0-5 $\mathrm{min}, 80 \%$ A 5-20 $\mathrm{min}, 80-40 \%$ A $20-30 \mathrm{~min}$, 40-0\% A 30-35 min. ${ }^{24,25}$ The injection volume was $5 \mu \mathrm{l}$. Detection wavelengths were set at 260 and $350 \mathrm{~nm}$, and the flow rate

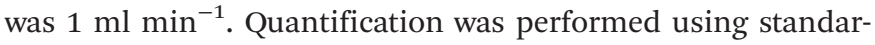
dized calibration curves of flavonoids (rutin, hyperoside and isoquercetin) and hydroxycinnamic acids (chlorogenic and neochlorogenic acids). The anthocyanin analysis utilized a mobile phase that consisted of solvent A (10\% of formic acid in water) and solvent B (acetonitrile) with an elution profile as follows: 99\% A 0-0.5 $\mathrm{min}, 99-93 \%$ A 1-4 $\mathrm{min}, 93-90 \%$ A 4-7 min, 90-86\% A 7-11 min, 86-75\% A 11-15 min, 75-60\% A 15-18 $\mathrm{min}, 60-25 \%$ A $18-22 \mathrm{~min}, 25 \%$ A $22-25 \mathrm{~min}$. Detection wavelengths were set at 290, 350 and $520 \mathrm{~nm}$, and the flow rate

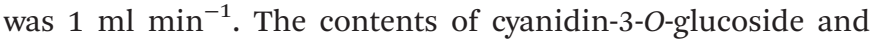
cyanidin-3-O-arabinoside were determined using calibration curves, while cyanidin-3-O-galactoside and cyanidin-3-O-xyloside were quantified in equivalents of cyanidin-3-O-arabinoside. All experiments were repeated at least two times. The results are presented as $\mathrm{mg} \mathrm{L}^{-1}$ of AJ.

Total proanthocyanidins were determined spectrophotometrically by a slightly modified vanillin assay. ${ }^{26}$ Namely, $20 \mu \mathrm{l}$ of the $2 \% \mathrm{AJ}$ in methanol in duplicate, or a range of concentrations of $(+)$-catechin in methanol, were mixed with $100 \mu \mathrm{l}$ of working vanillin reagent $(0.5 \%$ vanillin and $4 \% \mathrm{HCl}$ in methanol). After $20 \mathrm{~min}$ at $30{ }^{\circ} \mathrm{C}$, their absorbencies were measured at $500 \mathrm{~nm}(A 500)$. The contents of proanthocyanidins in AJ were expressed as $(+)$-catechin equivalents in $\mathrm{mg} \mathrm{L}^{-1}$.

\subsection{Animals and treatments}

Male adult Wistar rats, 4 months old, weighing 370-430 g, provided by the vivarium of the Institute for Biological Research, Belgrade, Serbia, were used in this study. The animals were kept in groups of $3-4$ per cage $(42 \times 26 \times$ $18 \mathrm{~cm}$ ), under controlled conditions (room temperature 23-25 ${ }^{\circ} \mathrm{C}, 12 \mathrm{~h}$ light-dark cycle, food and drinking solutions ad libitum). All animal procedures were in compliance with Directive 2010/63/EU on the protection of animals used for experimental and other scientific purposes. They were approved by the Ethical Committee for the Use of Laboratory Animals of the Institute for Biological Research "Siniša Stanković", University of Belgrade. 
Rats were randomly assigned to 3 experimental groups of 8 animals each, and one control group $(N=12)$. The experimental groups were supplied with different drinking solutions, prepared daily as combinations of AJ and RJ in tap water $(\mathrm{v} / \mathrm{v})$ : (1) ARO, contained 20\% AJ; (2) RAJ, 5\% AJ + 15\% RJ; (3) PLC, $20 \%$ RJ. The control group (CTL) consumed tap water. The rats were allowed to drink these solutions or water for the next 34 days, without restrictions. The amounts of the consumed drinking solutions and food were estimated daily per cage and calculated weekly per experimental group. Individual body masses, which had been measured before (Day-1) and at the end of the study (Day35), were used to determine the average gain of body mass $( \pm$ S.E.M.) for each experimental group. All animals were consuming commercial rat chow (Veterinarski zavod Subotica, Serbia; $20 \%$ of proteins). On the days: $-1,31$, 32 and 34, appropriate behavioral tests were performed ( $09 \mathrm{~h}-$ $13 \mathrm{~h}$ ), when rat activities were registered in a quiet room with shaded illumination, by video recording. Most of the behavioral parameters were detected and analyzed by ANY-maze Video tracking system (4.13; Stoelting, USA) while the remaining behavioral features were observed and recounted by a proficient examiner, who was unaware of group qualification of the rats. All parts of the apparatus that came in contact with the animals were cleaned with $10 \%$ alcohol at the end of each session.

\subsection{Behavioral tests}

2.4.1 Open field test (OFT). This test was used to estimate average ambulation of each rat on Day- 1 and Day31 of the study, when they were allowed to move freely in a plastic openbox $(44 \times 44 \times 35 \mathrm{~cm})$. The movements of single rats in four adjacent boxes were simultaneously registered by a PCconnected camera positioned centrally above, for $20 \mathrm{~min}$. The ANY-maze software evaluated animal activities according to two chosen parameters: (1) the measure of overall activity, presented by the percentage of total time when the animal was active, and (2) locomotion, by the distance traveled (in $\mathrm{m}$ ). ${ }^{27}$ The third analyzed parameter, (3) number of rearings (standing upright on the hind legs), which reflected vertical activity, was counted during observation of the video recordings.

2.4.2 Elevated plus maze (EPM). This is a typical test based on the innate fear of rodents of open and elevated spaces, which is applied to detect anxiety-like behaviors and to evaluate anxiolytic-like treatments in experimental animals. ${ }^{27}$ The EPM apparatus consisted of a cross-shaped Plexiglas platform with two opposite open arms (OA, $50 \times 10 \mathrm{~cm})$ and two opposite closed arms (CA, $50 \times 10 \mathrm{~cm})$ with $40 \mathrm{~cm}$ walls, connected by a central platform $(\mathrm{CP}, 10 \times 10 \mathrm{~cm})$ and elevated $50 \mathrm{~cm}$ from the floor. ${ }^{27}$ On the Day32, each rat was placed in a CP facing one of the CAs. Their movements were recorded for the next 5 min with a video camera mounted vertically above the apparatus. The level of rat anxiety was estimated by the periods spent in OA and number of entries into OA, which were quantified and presented in our test as \% of OA time and \% of OA entries. ${ }^{27,28}$ Another two parameters, the number of entries in CA and the time of immobility, were also used, as they may reflect general activity and ambulation. ${ }^{28,29}$ While these four parameters were estimated by ANY-maze software, the other two ethological parameters were detected and counted by an observer: the number of SAP (stretched attend posture; when a rat stretches its head and shoulders before returning to its initial position), which represented risk assessment, and the number of head dips (when a rat scans over the side of an OA towards the floor), as a reflection of exploratory behavior. ${ }^{28,30}$ A random half of CTL animals $(n=6)$, which were being supplied with pure drinking water, was considered as a primary control group with unmodified behavior (CTL). They received saline $(0.9 \% \mathrm{NaCl}) 1 \mathrm{ml}$ per $\mathrm{kg}$ of b. mass (ip), $60 \mathrm{~min}$ before the test, and the same was applied to all rats in the experimental groups. The other 6 CTL rats were treated with $1 \mathrm{mg} \mathrm{kg} \mathrm{kg}^{-1}$ of anxiolytic diazepam in saline, in the same manner as the other groups. They were considered as a reference treatment group with reduced anxiety-like behaviors (DZP).

2.4.3 Forced swimming test (FST). This test is based on the observations that rats forced to swim in an inescapable space express characteristic behavior of immobility, when they are floating in water and making only those movements that are necessary to keep their heads afloat. Such immobility reflects a state of despair or a depression-like form of behavior that can be reduced by human antidepressant drugs. ${ }^{27}$ Increases in active responses, such as climbing or swimming, and reduction in immobility, are considered as behavioral profiles consistent with an antidepressant-like action ${ }^{31}$ In this adaptation of the original FST, ${ }^{27,32}$ each rat was trained on Day33 for $12 \mathrm{~min}$, by being forced to swim in an open glass cylinder ( $R=20 \mathrm{~cm}, L=48 \mathrm{~cm}$ ), containing $25 \mathrm{~cm}$ of water maintained at $23-25{ }^{\circ} \mathrm{C}$. On Day34, rats were individually placed into the water tank for $6 \mathrm{~min}$ and their activities were registered from a vertical position by video camera. The active periods of swimming (defined as movement throughout the swim chamber) and climbing (upward-directed movements of the forepaws along the side of the swim chamber), together with the immobile-periods, were later observed, registered and summarized by a trained observer, using ANY-maze software. One hour before their individual test, a random 6 CTL rats and each rat of the 3 experimental groups were injected with saline (ip; $1 \mathrm{ml}$ per $\mathrm{kg}$ b. mass), while another $6 \mathrm{CTL}$ rats were injected with $15 \mathrm{mg} \mathrm{kg}^{-1}$ of antidepressant imipramine in the same manner. The first group represented the basic control group (CTL) and the second group was considered as the reference treatment group with reduced depression-like behaviors (IMI).

\subsection{MAO assays}

The effects of Aronia juice and phenolic compounds on in vitro MAO activity were evaluated using MAO-Glo Assay V1401 (Promega; USA) as described elsewhere. ${ }^{33}$ This two-step bioluminescent assay was performed in 96-well flat-bottom assay plates where the purified rat liver mitochondrial fraction was used as a source of MAO enzymes. ${ }^{34}$ In the first step, the mitochondrial microsomes were incubated with a substrate 
(concentrations were set at their $K_{\mathrm{m}}$ values of $40 \mu \mathrm{M}$ and $4 \mu \mathrm{M}$ for MAO-A and MAO-B, respectively) and various concentrations of the phenolic compounds $(0.1 \mu \mathrm{M}-100 \mu \mathrm{M} / 1 \mathrm{mM})$ or $\mathrm{AJ}$, for $1 \mathrm{~h}$ at room temperature. The dilutions of $\mathrm{AJ}$ in the assay were adjusted to the concentration of total phenolic compounds determined for AJ (6.484 $\left.\mathrm{g} \mathrm{L}^{-1}\right)$, so that their ranges of concentrations in the assay were $0.01-100 \mu \mathrm{g} \mathrm{mL} \mathrm{mL}^{-1}$. The reaction buffer for the MAO assay contained $100 \mathrm{mM}$ Hepes ( $\mathrm{pH} 7.5$ ) and 5\% glycerol, and for MAO-B, the buffer additionally contained 10\% dimethyl sulfoxide (DMSO), which was used to inhibit MAO-A and stimulate MAO-B activity. In the second step, after the addition of reconstituted luciferin detection reagent used to generate and stabilize the luminescence, the mixtures were incubated at room temperature for 20 minutes. Pargyline was used as a reference compound for both assays. The luminescent signal was measured using a Chameleon chemiluminescence reader (Hidex, Turku, Finland). The results were presented as $\mathrm{IC}_{50}$ values in $\mu \mathrm{g} \mathrm{mL}^{-1}$ of $\mathrm{AJ}$ phenolics, or in $\mu \mathrm{M}$ for the particular phenolic compounds.

\subsection{Data presentation and statistical analysis}

Experimental data are presented as mean \pm S.E.M., in relation to the number of animals. Statistical analyses of all experimental data, as well as the determination of $\mathrm{IC}_{50}$ values for MAO inhibition, were done by the Prism software (5.0; GraphPad software, USA). Intergroup differences were evaluated by either two-way (OFT) or one-way ANOVA, followed by NewmanKeul's post-hoc test for multiple comparisons (if $p<0.05$ ). Twoway Student's $t$-test was used to compare homologous Day31 and Day-1 values (OFT).

\section{Results}

\subsection{Characterization and quantification of phenolic constituents in $\mathrm{AJ}$}

The qualitative and quantitative analyses of $\mathrm{AJ}$ are presented in Table 1 . Only the identified phenolic compounds with concentrations $>10 \mathrm{mg} \mathrm{L}^{-1}$ are provided.

\subsection{Quantitative parameters of eating, drinking and body mass gain}

The average daily consumptions of drinking solutions in the experimental groups during this 5-week study are represented in Table 2. They are shown in $\mathrm{mL}$ per animal, instead of $\mathrm{ml}$ per body mass, considering the variable gains in body mass during the study. Analysis by two-way ANOVA revealed a significant influence of the week of the study $(F(4,120)=28.03, p<$ $0.0001)$ and of the contents of drinking solutions $(F(3,120)=$ $41.31, p<0.0001)$, but also of their interaction $(F(12,120)=$ $8.78, p<0.0001)$. These effects are reflections of the greatly enhanced drinking of solutions with Aronia phenolics, where only two groups supplemented with AJ showed significant elevations of daily drinking from the $2^{\text {nd }}(\mathrm{RAJ})$ or $3^{\text {rd }}$ week (ARO) onward. The consumed amount of the drinking solution in the ARO group was $50-70 \%$ higher vs. CTL, and about
Table 1 Quantities of phenolic compounds in Aronia melanocarpa fruit juice (AJ). Data expressed as mean values ( $n=2$ or 3 ), standard error $<8 \%$ of mean values

\begin{tabular}{|c|c|}
\hline Phenolic compounds & Amount $\left(\mathrm{mg} \mathrm{L}^{-1}\right)$ \\
\hline Total phenols ${ }^{a}$ & 6484 \\
\hline Total proanthocyanidins ${ }^{b}$ & 240 \\
\hline \multicolumn{2}{|l|}{ Phenolic acids $^{c}$} \\
\hline Chlorogenic acid & 1389 \\
\hline Neochlorogenic acid & 1057 \\
\hline \multicolumn{2}{|l|}{ Anthocyanins ${ }^{c}$} \\
\hline Cyanidin-3-O-glucoside & 21 \\
\hline Cyanidin-3-O-galactoside & 301 \\
\hline Cyanidin-3-O-arabinoside & 101 \\
\hline Cyanidin-3-O-xyloside & 13 \\
\hline \multicolumn{2}{|l|}{ Flavonols ${ }^{c}$} \\
\hline Quercetin-3-O-glucoside (isoquercetin) & 53 \\
\hline Quercetin-3-O-galactozide (hyperoside) & 97 \\
\hline Quercetin-3-O-rutinozide (rutin) & 194 \\
\hline \multicolumn{2}{|c|}{$\begin{array}{l}{ }^{a} \text { Folin-Ciocalteu method, concentration based upon gallic acid as } \\
\text { standard. }{ }^{b} \text { Determined spectrophotometrically by the vanillin assay, } \\
\text { concentration based upon (+)-catechin as standard. }{ }^{c} \text { Single com- } \\
\text { pounds determined by HPLC/DAD. The flavonoids (rutin, hyperoside } \\
\text { and isoquercetin), cyanidin-3-O-galactoside and hydroxycinnamic } \\
\text { acids (chlorogenic and neohlorogenic acids) were used as standards. }\end{array}$} \\
\hline
\end{tabular}

$40-60 \% v s$. PLC, throughout the $3^{\text {rd }}, 4^{\text {th }}$ and $5^{\text {th }}$ week, while this increase in the RAJ group went up to $30 \%$.

Table 3 shows the overall consumptions of the liquids and food in the experimental groups during the five weeks of the study. Also, this table provides the consumed quantities of the total phenolic compounds, proanthocyanidins and anthocyanins in $\mathrm{g}$ per $\mathrm{kg}$ of $\mathrm{b}$. mass in each experimental group. These calculations are based on the analytical data presented in Table 1 and the volumes of the solutions that were drunk weekly (Table 2). The volume-mass transformations are based on the mass densities of $1.10,1.09$ and $1.02 \mathrm{~g} \mathrm{~mL}^{-1}$, estimated for AJ, RJ and all drinking mixtures, respectively. Standard deviations of these values were not obtained since it was not possible to track individual consumptions within the group. The variations of the average rat chow consumptions among the groups were within $5 \%$, which may indicate that they are not significant. Otherwise, the amounts of the drinking solutions consumed in the groups RAJ and ARO were between 13\% and $30 \%$ higher than the amounts in groups CTL and PLC, which could be referred to as significant elevations. The calculated quantities of the phenolics that were consumed in the ARO group were about 4.5 times larger than in the RAJ group. Supplementary calculations for the period of the first 4 weeks of the study pointed out that the average daily phenolic consumptions per $\mathrm{kg}$ of rat body mass were: 30.8 and $133.0 \mathrm{mg}$ per kg per day in the RAJ and ARO groups, respectively. During the $5^{\text {th }}$ week of the study, when all behavioral tests were performed, these amounts were 33.0 (RAJ) and 168.0 (ARO) in mg per $\mathrm{kg}$ per day. The variations of the body mass gains among the groups were significant $[F(3,32)=5.535, p<0.0035$, oneway ANOVA], with conspicuous $60-70 \%$ enlargements in the ARO group when matched with the CTL and RAJ groups, but not in comparison with the PLC group. 
Table 2 Daily consumptions of drinking solutions in 5 weeks of the experiment

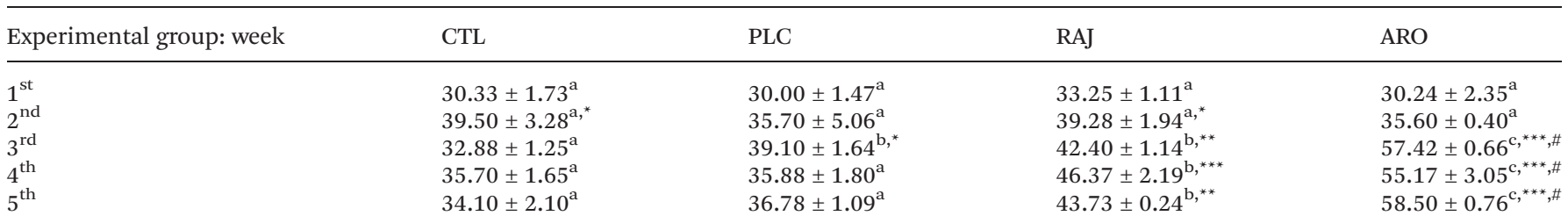

Experimental groups have been limitlessly supplied with tap water (CTL), and water dilutions of: 20\% RJ (PLC), 5\% AJ + 15\% RJ (RAJ), and 20\% AJ (ARO). Values of the average daily consumptions of drinking solutions (in ml per animal) for each of 5 weeks of the experiment are presented as mean \pm S.E.M., which reflected daily variations within a week $(N=7$ or 6$)$. Values with different letters in the same row are significantly different from each other $(p<0.05)$, while those that share the same letters are not $(p>0.05)$. The column statistics is presented with symbols: ${ }^{*}, p<0.05 ;{ }^{* *}, p<0.01 ;{ }^{* * *}, p<0.001 v s .1$ st week. ${ }^{\#}, p<0.001 v s .2$ nd week. Newman-Keuls multiple comparisons test was applied after two-way ANOVA.

Table 3 Cumulative consumptions of drinking solutions, AJ, total phenolics, proanthocyanidins, anthocyanins and rat chow, and average rat body mass gains in the experimental groups

\begin{tabular}{lllll}
\hline Experimental group: & \multicolumn{1}{c}{ CTL } & PLC & RAJ & ARO \\
\hline Consumption (g per kg b. mass) of: & & & \\
Drinking solution & 2813 & 2906 & 3273 & 3645 \\
AJ & 0 & 0 & 176 & 786 \\
Phenolics & 0 & 0 & 1.037 & 4.636 \\
Proanthocyanidins & 0 & 0 & 0.039 & 0.172 \\
Anthocyanins & 0 & 0 & 0.071 & 0.315 \\
Rat chow & 1775 & 1817 & 1718 & 1731 \\
Body mass gain & $4.8 \pm 0.5$ & $6.9 \pm 0.6^{*}$ & $5.1 \pm 0.7$ & $8.1 \pm 0.9^{*}$,\# \\
(\% of b. mass on Day-1) & & & &
\end{tabular}

Overall consumptions throughout 34 days of the experiment are presented for the rat chow and for each of the drinking solutions, where the consumed amounts of these solutions, AJ, total phenolics, proanthocyanidins and anthocyanins of AJ, are shown in grams per kg of average body mass [(Day-1 + Day35)/2]. Mass densities of AJ and RJ were 1.10 and $1.09 \mathrm{~g} \mathrm{~mL}^{-1}$, respectively, while they were $1.02 \mathrm{~g} \mathrm{~mL}^{-1}$ for all drinking solutions except tap water. Average body mass gains in each experimental group was estimated on Day35 and presented as percentage of body mass on Day- $1 \pm$ S.E.M. $(N=8$, or $N=12$ for CTL $)$ ${ }^{*}, p<0.05$ vs. CTL; \#, $p<0.05$ vs. RAJ by post hoc Newman-Keuls multiple comparisons test.

\subsection{Open field test}

Two of the parameters evaluated by OFT before the start of the study, the distance traveled (Fig. 1A) and the active time (Fig. 1B), did not show significant variations among the experi- mental groups $[F(3,32)=0.901, P=0.451$ and $F(3,32)=1.309$, $P=0.288$, respectively], while the influences of the one-month treatments on these parameters were evident on Day31 $[F(3,32)$ $=3.917, P=0.017$ and $F(3,32)=2.924, P=0.0488$, respectively]. The post-hoc test revealed significant differences between the groups: ARO and CTL $(+35 \%, p<0.05)$, as well as for ARO vs. RAJ ( $+46 \%, p<0.05)$, regarding the distance traveled. Also, active time was enhanced in the ARO group in comparison with both the CTL $(+33 \%, p<0.05)$ and RAJ $(+32 \%, p<0.05)$ groups. The fluctuation of these parameters in the same experimental group between Day31 and Day-1 were verified by the Student's $t$-test, which revealed significant increases of both distances traveled $(+22 \%, p=0.040$; Fig. 1A) and active times $(+20 \%, p=0.032$; Fig. $1 \mathrm{~B})$ only in the ARO group. The rearings recorded in OFT (Fig. 1C) were not significantly altered on both Day-1 and Day31 $[F(3,32)=1.231, P=0.315$ and $F(3,32)=1.139, P=0.348$, respectively], but there were almost significant elevations of this parameter in the RAJ $(+56 \%, p=0.053)$ and ARO $(+40 \%, p=0.059)$ groups, between Day31 and Day-1.

\subsection{Elevated plus maze}

EPM was used on Day32 of the experiment to determine the effects of treatments on the anxiety-like forms of rat behavior (Fig. 2). Two parameters of EPM that reflect the opposite to such behavior were significantly altered: \% of time spent in OA $(F(4,31)=5.874, p=0.0012$; Fig. $2 \mathrm{~A})$, with a significant
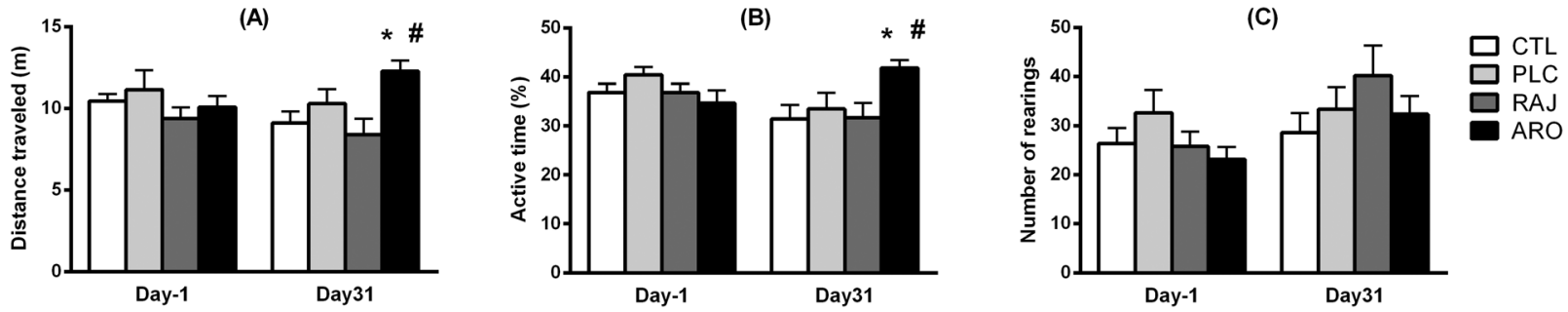

Fig. 1 Effects of month-long unlimited drinking of either tap water (CTL), 20\% RJ (PLC), 5\% AJ + 15\% RJ (RAJ), or 20\% AJ (ARO), on rat behavior in OFT. The overall rat activities were registered during $20 \mathrm{~min}$, before the start of the experiment (Day-1) and on Day31 of the study. Three OFT parameters, evaluated by ANY-maze software, are presented: (a) distance traveled, (b) percentage of active time, and (c) number of rearings, which are expressed as group mean \pm S.E.M. values ( $n=12$ or 8 ). ${ }^{*} p<0.05$, vs. CTL, by one-way ANOVA post hoc Newman-Keuls multiple comparison test. $\# p<0.05$, by two-way student's $t$-test, Day31 vs. Day-1. 

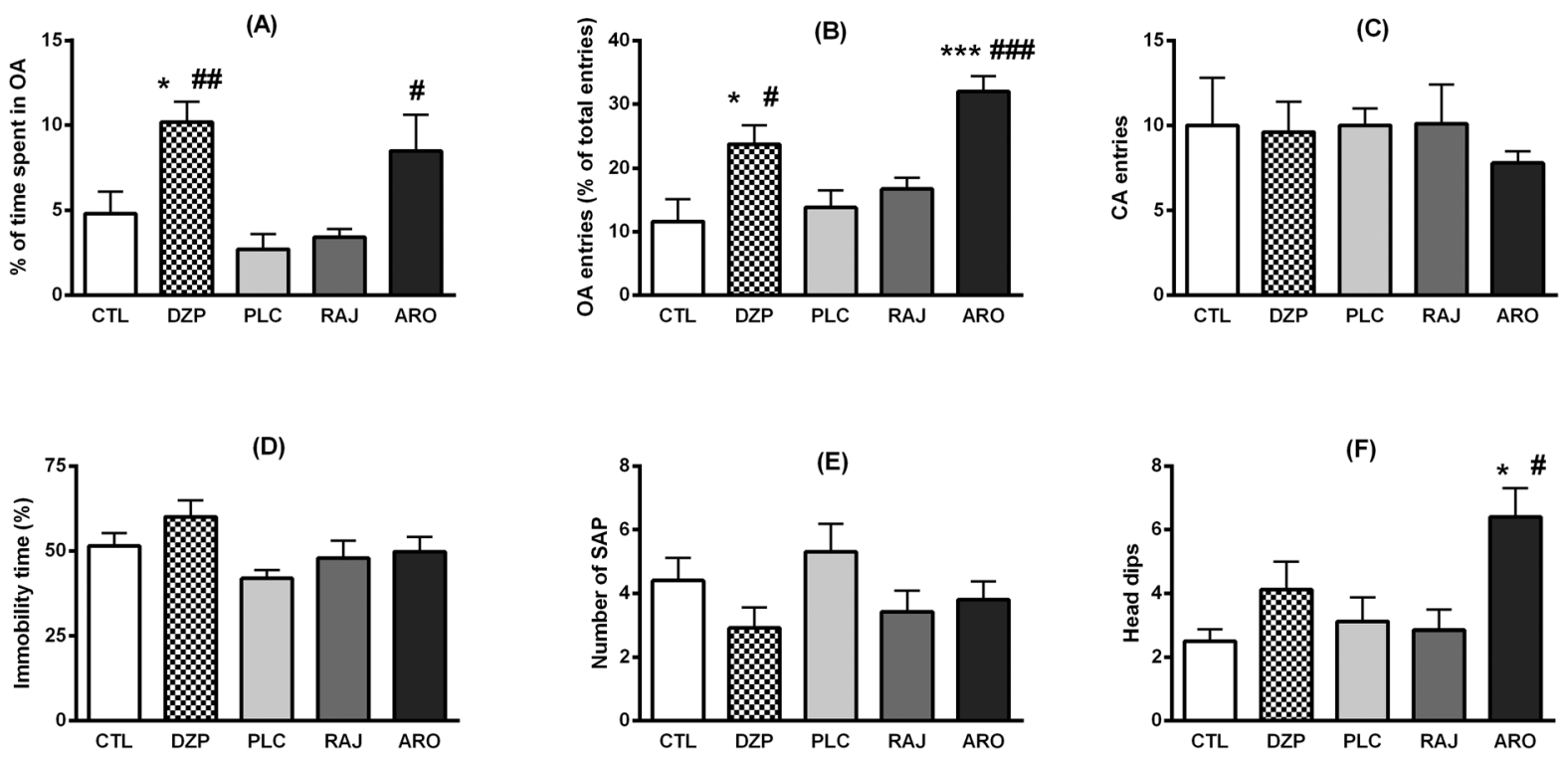

Fig. 2 Effects of month-long unlimited drinking of the experimental mixtures (details about the experimental groups are in the caption of Fig. 1) on rat behavior in EPM. Each rat had been injected (ip; $1 \mathrm{ml}$ per $\mathrm{kg} \mathrm{b}$. mass), one hour before the test was performed on Day32, with either 1 mg kg ${ }^{-1}$ diazepam (DZP; random 6 of 12 rats scheduled to drink only tap water) or saline (all other rats in the experimental groups and CTL). The rat behavioral parameters in EPM (as mean \pm S.E.M. $n=6$ or 8 ) were recorded for 5 min and evaluated with ANY-maze software or by observation, (a) percentage of time spent in OA, (b) percentage of OA entries, (c) CA entries, (d) percentage of immobility time, (e) number of stretched attend postures (SAP)

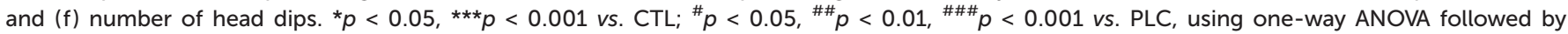
Newman-Keuls post-hoc tests.

increase in ARO vs. PLC $(+215 \% ; p<0.05)$ and $v s . \mathrm{RAJ}(+150 \%$; $p<0.05)$, but not vs. CTL $(+77 \% ; p>0.05)$, and $\%$ of $\mathrm{OA}$ entries $(F(4,31)=10.4, p<0.0001$; Fig. $2 \mathrm{~B})$, which was more strongly expressed in the ARO group $(+176 \%$ vs. CTL, and +132 vs. PLC; $p<0.001)$ than in the DZP group $(+105 \% v s$. CTL, and +72 vs. PLC; $p<0.05)$. Two parameters of EPM that may reflect general activity and ambulation, the number of entries in CA (Fig. 3C) and the time of immobility (Fig. 3D), were not substantially affected by the treatments $(F(4,31)=$ $0.321, p=0.861$ and $F(4,31)=2.307, p=0.080$, respectively). It was the same with the number of SAP (Fig. 3E), which represented risk assessment $(F(4,31)=0.321, p=0.861)$. On the contrary, the number of head dips (Fig. 3F), as a reflection of exploratory behavior, was clearly influenced by the treatments $(F(4,31)=4.584, p=0.005)$, with significant elevations in the ARO group vs. the CTL $(+156 \% ; p<0.05)$, PLC $(+105 \% ; p<$ $0.05)$ and RAJ $(+125 \% ; p<0.05)$ groups.

\subsection{Forced swimming test}

This test was used as a regular model for screening the antidepressant-like action of the treatments. The percentages of time when animals were immobile during 6 min in the cylindrical water container, which represented a depression-like form of behavior on Day34, are shown in Fig. 3A. There was a very significant effect of the treatments on the immobile periods in the FST $(F(4,31)=11.57, p<0.0001)$. The post-hoc test revealed that this parameter was significantly diminished in both the ARO $(-28 \%$ vs. CTL, and $-23 \%$ vs. PLC; $p<0.001)$ and IMI $(-21 \%$ vs. CTL, and $-16 \%$ vs. PLC; $p<0.01)$ group. The influence of the treatments on the periods of active swimming, which may be interpreted as the modulation of seroto$\operatorname{nin}(5-\mathrm{HT})$ neurotransmission, ${ }^{32}$ also appeared to be very significant $(F(4,31)=21.15, p<0.0001$; Fig. 3B). This was an outcome of exceptionally intensified swimming in the ARO group $(+133 \%$ vs. CTL, and $+93 \%$ vs. PLC; $p<0.001)$, and of a lesser swimming increase in the IMI group $(+61 \%$ vs. CTL, and $+33 \%$ vs. PLC; $p<0.05)$. There was a modest effect of the treatments on climbing behavior $(F(4,31)=3.90, p<0.011$; Fig $3 \mathrm{C})$, which indicated the levels of noradrenaline (NE) neurotransmission, ${ }^{32}$ whereas only the rats that received imipramine exhibited an increase in this parameter $(p<0.05$, IMI vs. CTL or PLC).

\subsection{MAO assays}

The abilities of AJ to inhibit MAO-A or MAO-B enzymes were evaluated by the MAO-Glo Assays (Table 4). The $\mathrm{IC}_{50}$ values (50\% inhibitory concentration of the phenolic constituents in $\mu \mathrm{g} \mathrm{L}^{-1}$ ) were calculated in relation to the determined $\mathrm{AJ}$ concentration of the phenolics $(0.65 \%)$. These data were compared with the estimated $\mathrm{IC}_{50}$ values (in $\mu \mathrm{M}$ ) for some of the phenolic compounds that had been detected in AJ, and pargyline, as a referent MAO inhibitor. The MAO inhibiting potency of AJ was more prominent for MAO-A than for MAO-B enzymes, while quercetin was the only one among the tested compounds that showed substantial MAO-A inhibition (Table 4). 

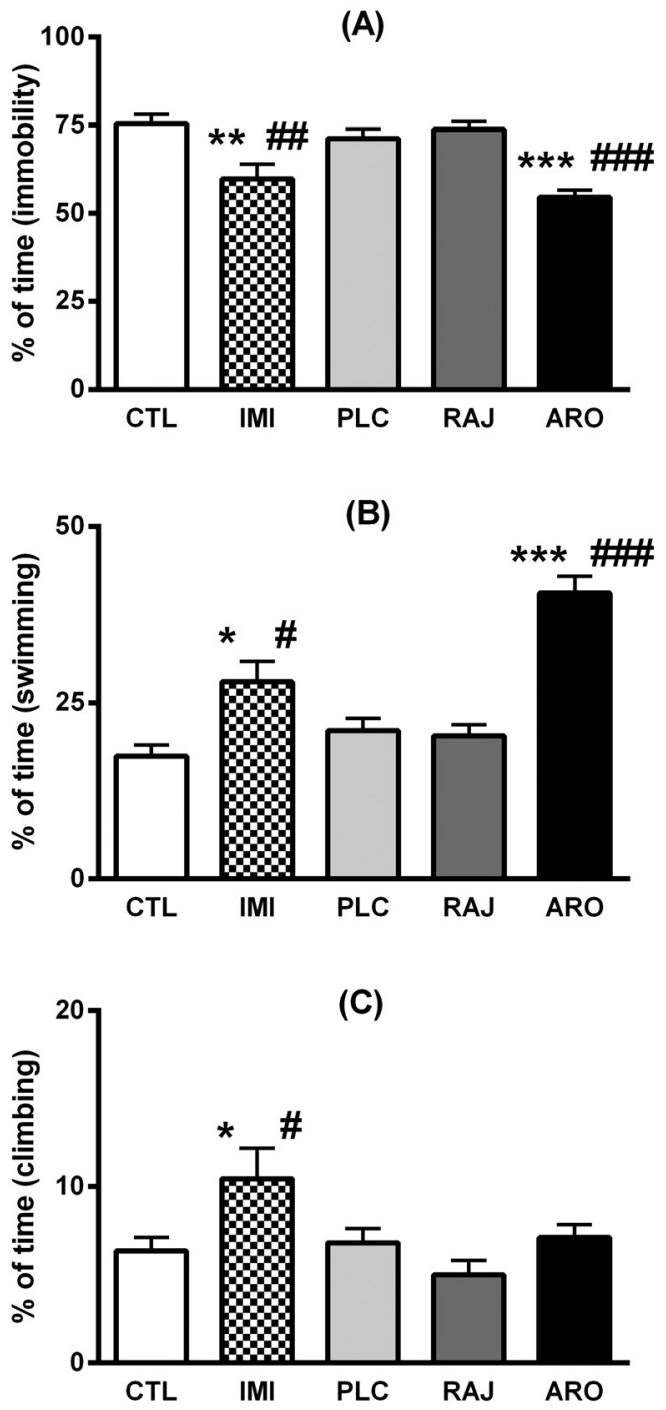

Fig. 3 The influence of month-long free drinking of the experimental mixtures on the proportion of the periods of (a) immobility, (b) active swimming, and (c) climbing, during $6 \mathrm{~min}$ in FST. (The same experimental groups are presented as in Fig. 1 and 2.) One hour before the test was performed on Day34, rats had been injected (ip; $1 \mathrm{ml}$ per $\mathrm{kg}$ b. mass) with either $15 \mathrm{mg} \mathrm{kg}^{-1}$ imipramine (IMI; random 6 of 12 rats on water consumption) or saline (all other rats of CTL and the experimental groups). Results are expressed as mean \pm S.E.M. ( $n=6$ or 8 ). ${ }^{*} p<0.05$, ${ }^{* *} p<0.01,{ }^{* * *} p<0.001$ vs. CTL; ${ }^{\#} p<0.05,{ }^{\# \#} p<0.01,{ }^{\# \# \#} p<0.001$ vs. PLC using one-way ANOVA followed by Newman-Keuls post-hoc tests.

\section{Discussion}

The results of this study demonstrate that month-long consumption of diluted AJ with a high level of phenolic compounds may induce significant behavioral variations in male rats, which were expressed as somewhat increased locomotion and reduced anxiety-like and depression-like forms of behavior. These behavioral modifications are related to unlimited drinking of $20 \% \mathrm{AJ}$ water dilution during one month (Table 2). The concept of this study was quite different from the typical nutritional-behavioral experimental studies, where specific
Table 4 In vitro MAO-A and MAO-B inhibition by $\mathrm{AJ}$ and the specific phenolic compounds

\begin{tabular}{|c|c|c|}
\hline Compounds & $\mathrm{IC}_{50} \mathrm{MAO} \mathrm{A}^{a}$ & $\mathrm{IC}_{50} \mathrm{MAO} \mathrm{B}{ }^{a}$ \\
\hline $\mathrm{AJ}\left(\mu \mathrm{g} \mathrm{L^{-1 }}\right)^{b}$ & 2.2 & 58.4 \\
\hline Chlorogenic acid $(\mu \mathrm{M})$ & $\mathrm{ND}^{c}$ & $\mathrm{ND}^{c}$ \\
\hline Cyanidin-3-O-glucoside $(\mu \mathrm{M})$ & 96.3 & 209 \\
\hline Cyanidin-3-O-arabinoside $(\mu \mathrm{M})$ & 80.8 & 477 \\
\hline Quercetin $(\mu \mathrm{M})$ & 0.72 & 170 \\
\hline Rutin $(\mu \mathrm{M})$ & 260 & 105 \\
\hline$(+)$-Catechin $(\mu \mathrm{M})$ & 38.5 & 22.0 \\
\hline Pargyline $(\mu \mathrm{M})^{d}$ & 6.7 & 0.22 \\
\hline
\end{tabular}

${ }^{a}$ Each inhibitory activity is expressed as the mean of $50 \%$ inhibitory concentration $\left(\mathrm{IC}_{50}\right)$ of two experiments, which was obtained by an interpolation of the concentration-inhibition curves. ${ }^{b}$ Adjusted to the concentration of phenolics in AJ, determined by Folin-Ciocalteu method. ${ }^{c}$ Not determined as the significant inhibition $(>50 \%)$ for the concentrations of up to $1 \mathrm{mM}$. ${ }^{d}$ Used as a comparative MAO inhibitor.

diets, rich in active ingredients, or purified natural compounds, are provided to animals by monitored oral administrations. The approach applied here allowed the animals to control their drinking habits in line with their general behavior, which was analogous to human living patterns. Accordingly, it was found that the adjunction of AJ in drinking water induced very significant elevations of the daily drinking amounts, from the $2^{\text {nd }} / 3^{\text {rd }}$ week onward, which was more pronounced in the ARO than in the RAJ group (Table 2). Only the average group consumption of either mixture was being tracked here, since it was not possible to control and determine individual drinking amounts. The progressive consumptions of both drinking mixtures with AJ over time revealed that the rats developed increased affinity for this juice starting from the $2^{\text {nd }} / 3^{\text {rd }}$ week of the experiment. A possible explanation for this may be increased thirst, which could be induced by the bitter-astringent taste of $\mathrm{AJ}^{35}$ However, the main source of bitterness in AJ should be amygdalin, ${ }^{9}$ which has been reported to have thirst-quenching capabilities. ${ }^{36}$ Moreover, in our pilot test, which was performed on 5 rats that were allowed to drink $20 \%$ mixture of $\mathrm{AJ}$ in water for 20 days, the average $30 \%$-increment of the solution volume that was drunk on Day20 (vs. Day-1), was completely reduced on Day21 $(+2 \%$ vs. Day-1), when the rats were supplied with water only (data unpublished). This information should not exclude a temporary induction of thirst by sustained AJ consumption, whereby the increased affinity for the particular component(s) of Aronia juice may be another option.

The dietary effects of intensified drinking in this study seem to promote body mass gain only for the ARO rats, when compared to the CTL rats. It may be supposed that the highest amount of the drinking solution that was drunk by the ARO rats $(+11 \%$ vs. RAJ and $+25 \%$ vs. PLC) was the reason for their body mass gain, since the consumption of rat chow was not enhanced in this group (Table 3). On the other hand, the PLC group showed certain elevations in both the rat chow eating and body mass gain (Table 3). A similar dietary study, where 
Aronia extract did not alter food intake and water consumption in rats that were fed a fructose-reach diet during 6 weeks while their body mass gain and epididymal adipose weight were reduced, suggests that Aronia might be beneficial in preventing or decreasing obesity and the metabolic syndrome. ${ }^{37}$ Another nutritional experiment showed a slight decrease in diet utilization and growth for the rats on Aronia polyphenolic diets relative to the control group. ${ }^{38}$ In an analogous human study, when $100 \mathrm{ml}$ of polyphenol-rich Aronia juice was consumed by healthy women, they showed no changes in body weight or in habitual dietary intake after 3 months on this regimen. ${ }^{39}$ The discrepancies in the registered dietary effects of Aronia between these and our study are coherent, having in mind the different concepts related to unlimited $v s$. restricted consumptions of Aronia products. Taking all this into account, it seems that the registered increase in affinity for the diluted AJ and its extensive consumption solely contributed to the enhanced body mass gains of the ARO rats, since this research did not show that the free drinking of Aronia juice could stimulate appetite and food intake.

The behavioral tests in this study were performed after the one-month drinking regimen, during 4 consecutive days (31-34). ARO was the only experimental group with significant behavioral variations in this period. These effects should be the result of drinking large amounts of phenolic-rich $\mathrm{AJ}$, where the calculated average amount consumed daily in the first 4 weeks was about $22 \mathrm{~g}$ per $\mathrm{kg}$ b. mass (133 $\mathrm{mg}$ per $\mathrm{kg}$ per daily of phenolic constituents). During the $5^{\text {th }}$ week, when the behavioral tests were performed, the ARO rats took over $30 \mathrm{~g} \mathrm{~kg}^{-1}$ of AJ daily (168 mg per kg per daily of phenolics), which was about 5 times the AJ amount consumed in the RAJ group ( $6 \mathrm{~g}$ $\mathrm{kg}^{-1}$ ). The behavioral consequences, registered by OFT in the ARO group on Day31, were increased field activity and locomotion in relation to both the Day-1 and CTL group (Fig. 1A and $\mathrm{B}$ ), while the multiplication of rearings was almost significant $(+40 \%$ vs. Day-1, $p=0.059$; Fig. $1 \mathrm{C})$. On the other hand, these parameters of activity were not significantly higher in the ARO vs. PLC group (Fig. 1), which suggested that the phenolic compounds were not solely responsible for that effect. A comparative analysis of the results generated by OFT and EPM indicates that the supposed hyperactivity of the ARO group may be alternatively explained as an expression of the exploratory behaviors, which could be reinforced by the reduction of innate anxiety. This assumption has its roots in the facts that no hyperactivity of the ARO group was found in EPM (evaluated by the number of CA entries and the percentage of immobility time; Fig. $2 \mathrm{C}$ and $\mathrm{D}$ ), while an elevation of the parameters that are opposite to the anxiety-like behaviors (the percentage of time spent in OA, the percentage of OA entries; Fig. 2A and B) come together with an increased number of head dips in the ARO group (Fig. 2F), which reflected exploratory behaviors. ${ }^{28}$ The enhancements of these three EPM parameters in the ARO group were exhibited toward three other experimental groups (CTL, PLC and RAJ), whilst they were similar to the acute effects of the anxiolytic diazepam (DZP; $1 \mathrm{mg} \mathrm{kg}^{-1}$ ). This evidence implies that only the intensive every- day consumption of AJ may induce anxiolytic-like effects in rats, which are detectable by EPM. The results of FST, presenting the reduction of immobile periods in ARO in comparison to the other three groups (Fig. 3A), which was more convincing than in the group acutely treated with the antidepressant imipramine (IMI; $15 \mathrm{mg} \mathrm{kg}^{-1}$ ), suggest that everyday consumption of AJ may also elicit antidepressant-like effects in rats. Since it has been shown that the antidepressant-like actions in FST, which were mediated by the stimulation of 5-HT neurotransmission, induced the increase of active swimming period, whereas the augmentation of NE neurotransmission was related to the enhanced climbing activity, ${ }^{32}$ the here registered activation of active swimming, but not of climbing in the ARO group (Fig. 3B and C), suggests that this antidepressant-like effect in rats may proceed from the stimulation of the neurotransmission mediated by 5-HT and not by NE. The absence of both the increased climbing in FST and rat hyperactivity in EPM may testify against a possibility that the OFT-referred ambulatory stimulation in ARO could simulate anti-depressant like effect in FST. Nevertheless, to clearly disprove (or not) this plausibility, more sensitive and distinctive behavioral evaluation of the antidepressant effect must be performed in the further research on Aronia extracts. In any case, such reductions of the anxiety-like and depression-like behaviors, and a possible psycho-stimulation in animals, which followed a sustained AJ consumption, may be interpreted as beneficial from the human viewpoints. ${ }^{40,41}$

A brief glance at reviews in biomedical literature reveals a substantial number of studies presenting the effects of natural phenolic-rich sources, mainly of the flavonoid group, on the behavior of experimental animals and humans. ${ }^{42,43}$ Hence, there are only several studies where Aronia juices/extracts were investigated in such a manner. The group of Valcheva-Kuzmanova found by passive avoidance tests that one-month consumption of Aronia juice (up to $10 \mathrm{ml}$ per $\mathrm{kg}$ b. mass daily) could improve memory in rats, while the social interaction test suggested some anxiolytic-like effects. ${ }^{16,17}$ However, they did not find any effects on locomotion in OFT. ${ }^{16}$ This may be in line with our evidence, where the RAJ group, with an approximate daily consumption of $\mathrm{AJ}$ in the range $5-6 \mathrm{ml} \mathrm{kg}^{-1}$, was not overactive. The ARO group, which showed increased locomotion in our study, received more than $20 \mathrm{ml} \mathrm{kg}^{-1}$ of AJ daily. Experiments with other polyphenol-rich fruit extracts showed particular behavioral and cognitive effects. It has been reported that low-molecular proanthocyanidins from grapes, applied to mice for 7 days in doses of up to $50 \mathrm{mg} \mathrm{kg}^{-1}$, exerted antidepressant-like effects in behavioral despair tests but did not affect locomotor activity. ${ }^{19}$ The proposed antidepressant mechanism was dose-dependent inhibition of MAO-A, and, to a lesser extent, of MAO-B, which induced marked increases of serotonin, as well as of dopamine and noradrenaline, in the frontal cortex, hippocampus and hypothalamus. ${ }^{19}$ Another study using in vitro MAO assays did not find significant effects of proanthocyanidins B1 and B2, but revealed that anthocyanidins and anthocyanidin-3-glycosides inhibited the activities of both MAO-A and MAO-B in the low 
micromolar range. ${ }^{20}$ The well-known medical applications of MAO-A inhibitors as antidepressants and, in drug combinations, as anti-anxiety drugs, ${ }^{44}$ combined with growing evidence that flavonoids may inhibit MAO enzymes, ${ }^{45}$ has motivated us to evaluate AJ and some of its phenolic constituents by in vitro MAO assays (Table 4). These assays revealed reliable MAO-A inhibition by $\mathrm{AJ}\left(\mathrm{IC}_{50}=2.2 \mu \mathrm{g}\right.$ of Aronia phenolics per L), which was expressed in relation to the determined concentration of AJ phenolic compounds (6.48 $\left.\mathrm{g} \mathrm{L}^{-1}\right)$. The reconstituted phenolic-free Aronia juice, RJ, did not affect MAO enzymes in the assay (data not included in Table 4). The levels of MAO inhibition, generated by the specific phenolic compounds in these assays, mostly corresponded to the literature data $^{20,45,46}$ where quercetin appeared to be the most potent MAO-A inhibitor $\left(\mathrm{IC}_{50}=0.72 \mu \mathrm{M}\right)$. However, this flavonol was found as aglycon only in traces in AJ, while the most abundant phenolics in AJ showed poor or insignificant interactions with MAO enzymes (Table 4). Obviously, it is challenging to estimate the participation of individual phenolic compounds in the resulting MAO inhibiting potency of AJ, especially with a reasonable presumption that there was a synergistic action of the various phenolics. Also, there is the question of the exact AJ phenolic composition that was being consumed throughout the study, knowing that the qualitative/quantitative analyses had been performed on fresh juice, before the start of the experiment. Considering both, the evidence that anthocyanin stability in commercial Aronia juices was satisfactory $\left(t_{1 / 2}=6-7\right.$ weeks at $21^{\circ} \mathrm{C}, 30-50$ weeks at $\left.4{ }^{\circ} \mathrm{C}\right),{ }^{47}$ while all other phenolic compounds in Aronia juices were found to be much more stable than anthocyanins, ${ }^{48}$ together with the duration of our experiment and the handling of $\mathrm{AJ}$, the stable concentrations of AJ phenolics is prospective throughout this study.

The greatest challenge is to extrapolate these in vitro data to the complexity of a living organism. Dietary polyphenols are highly sensitive to the mild alkaline conditions in the small intestine and a good proportion of these compounds can be transformed into other unknown and/or undetected structural forms with different chemical properties and, consequently, different bioaccessibility, bioavailability and biological activity. ${ }^{49}$ Anthocyanins are poorly absorbed in the small intestine, so they are recovered in blood and found in urine in nanomolar concentrations, with the concern that conjugation (glucuronidation and methylation) affects the biological activity of anthocyanins in vivo. ${ }^{10,50}$ Proanthocyanidin particles that may be absorbed through the gut barrier can be up to trimer size and they constitute less than 5\% of their total amount. ${ }^{10}$ So, these two groups of polyphenols which are present in moderate amounts in AJ are the least well absorbed. Chlorogenic and neochlorogenic acids are found in significant concentrations in Aronia, but esterification decreases their intestinal absorption. ${ }^{10}$ Monomeric flavonols and flavan-3-ols, like those usually detected in Aronia ((-)-epicatechin and quercetin) are more efficiently absorbed than anthocyanins, but their amounts in $\mathrm{AJ}$ are very low and it is not very likely that they have a significant influence on plasma polyphenol concentrations. ${ }^{10,49}$ On the other hand, quercetin glucosides, such as isoquercetin, have been shown to be much more quickly absorbed and more bioavailable than the smaller quercetin aglycone, or the larger quercetin glycosides such as rutin. ${ }^{51,52}$ The findings that glucoside chains are submitted to highly effective hydrolysis in the small intestine endothelium indicate that most of the ingested flavanols may enter circulation as quercetin aglycones. ${ }^{52}$ So the therapeutic profiles of all flavanols found in Aronia may correspond to that of quercetin. Another and still open query is related to the brain bioavailability of polyphenols, dependent on their passing through the blood-brain barrier, ${ }^{53}$ which is, however, beyond the scope of this study. At all events, it is plausible that the combinations of the original and transformed phenolic compounds of $\mathrm{AJ}$ may attain rat circulation and pass the blood-brain barrier in such concentrations that they could produce certain neurochemical effects by synergism. A part of these effects would be achieved by the central MAO inhibition (especially of MAO-A). It may result preferentially in the up-regulation of central serotonin and not of noradrenaline levels. Furthermore, the previously registered direct influences of particular bioflavonoids on the neurotransmitter reuptake processes, ${ }^{18,21}$ GABA receptors, ${ }^{45}$ even on acetyl-cholinesterase, ${ }^{54}$ suggest that these additional interactions may contribute to the behavioral alterations observed in ARO rats in this study. Only further investigations may answer some of the questions regarding the exact central influence of the phenolic constituents of Aronia.

\section{Conclusions}

The present findings demonstrate significant behavioral variations in male rats after one month of unlimited drinking of diluted AJ (20\%), when the average daily consumption of Aronia phenolic compounds exceeded $130 \mathrm{mg}$ per $\mathrm{kg}$ b. mass. These changes were registered in animal models of behavior and recognized as somewhat increased locomotion and the reduction of anxiety-like and depression-like forms of behavior. Although this study suggests that the central MAO-A inhibition by the Aronia phenolic compounds may be important for such behavioral effects, further research would shed more light on these interactions.

\section{Acknowledgements}

This work was supported by the Ministry of Education, Science and Technological Development of Republic of Serbia, grant III 41030.

\section{References}

1 R. Whitaker, Ethical Hum. Psychol. Psychiatry, 2005, 7, 23-35.

2 H. P. N. (SMA) 14-4887 NSDUH Series H-49, Results from the 2013 National Survey on Drug Use and Health, Mental Health Findings, Rockville, MD, 2014. 
3 D. Souery, G. I. Papakostas and M. H. Trivedi, J. Clin. Psychiatry, 2006, 67(Suppl. 6), 16-22.

4 L. N. Ravindran and M. B. Stein, J. Clin. Psychiatry, 2010, 71, 839-54.

5 N. Iovieno, E. D. Dalton, M. Fava and D. Mischoulon, J. Affective Disord., 2011, 130, 343-57.

6 S. N. Young, J. Psychiatry Neurosci., 1993, 18, 235-44.

7 A. Wilczynska, R. B. Singh and F. De Meester, Open Nutraceuticals J., 2011, 4, 52-60.

8 L. M. Manosso, M. Moretti and A. L. Rodrigues, Food Funct., 2013, 4, 1776-1793.

9 S. Kulling and H. Rawel, Planta Med., 2008, 74, 1625-1634.

10 P. N. Denev, C. G. Kratchanov, M. Ciz, A. Lojek and M. G. Kratchanova, Compr. Rev. Food Sci. Food Saf., 2012, 11, 471-489.

11 A. W. Strigl, E. Leitner and W. Pfannhauser, Z. Lebensm.Unters. Forsch., 1995, 201, 266-268.

12 X. Wu, L. Gu, R. L. Prior and S. McKay, J. Agric. Food Chem., 2004, 52, 7846-7856.

13 C. Chrubasik, G. Li and S. Chrubasik, Phytother. Res., 2010, 24, 1107-1114.

14 J. Kong, Phytochemistry, 2003, 64, 923-933.

15 A. Kokotkiewicz, Z. Jaremicz and M. Luczkiewicz, J. Med. Food, 2010, 13, 255-269.

16 S. Valcheva-Kuzmanova and M. Zhelyazkova-Savova, Methods Find. Exp. Clin. Pharmacol., 2009, 31, 651-654.

17 S. V. Valcheva-Kuzmanova, M. T. Eftimov, R. E. Tashev, I. P. Belcheva and S. P. Belcheva, Folia Med., 2014, 56, 199203.

18 Y. Xu, Z. Wang, W. You, X. Zhang, S. Li, P. A. Barish, M. M. Vernon, X. Du, G. Li, J. Pan and W. O. Ogle, Eur. Neuropsychopharmacol., 2010, 20, 405-13.

19 Y. Xu, S. Li, R. Chen, G. Li, P. A. Barish, W. You, L. Chen, M. Lin, B. Ku, J. Pan and W. O. Ogle, Pharmacol., Biochem. Behav., 2010, 94, 447-53.

20 A. Dreiseitel, G. Korte, P. Schreier, A. Oehme, S. Locher, M. Domani, G. Hajak and P. G. Sand, Pharmacol. Res., 2009, 59, 306-11.

21 M. Yáñez, N. Fraiz, E. Cano and F. Orallo, Biochem. Biophys. Res. Commun., 2006, 344, 688-95.

22 P. G. Waterman and S. Mole, Analysis of phenolic plant metabolites, Blackwell Scientific, Oxford, Boston, 1994.

23 N. Stanisavljevic, J. Samardzic, T. Jankovic, K. Šavikin, M. Mojsin, V. Topalovic and M. Stevanovic, Food Chem., 2015, 175, 516-522.

24 N. Ćujić, K. Šavikin, T. Janković, D. Pljevljakušić, G. Zdunić and S. Ibrić, Food Chem., 2016, 194, 135-142.

25 K. Šavikin, G. Zdunić, T. Janković, D. Gođevac, T. Stanojković and D. Pljevljakušić, Food Chem., 2014, 62, 677-683.

26 M. L. Price, S. Van Scoyoc and L. G. Butler, J. Agric. Food Chem., 1978, 26, 1214-1218.

27 Drug discovery and evaluation: pharmacological assays, ed. H. G. Vogel, Springer-Verlag, Berlin, Heidelberg, 2nd edn, 2002.
28 R. J. Rodgers, B.-J. Cao, A. Dalvi and A. Holmes, Braz. J. Med. Biol. Res., 1997, 30, 289-304.

29 A. Cruz, F. Frei and F. Graeff, Pharmacol., Biochem. Behav., 1994, 49, 171-176.

30 V. Carola, F. D’Olimpio, E. Brunamonti, F. Mangia and P. Renzi, Behav. Brain Res., 2002, 134, 49-57.

31 J. F. Cryan, A. Markou and I. Lucki, Trends Pharmacol. Sci., 2002, 23, 238-245.

32 M. J. Detke, M. Rickels and I. Lucki, Psychopharmacology, 1995, 121, 66-72.

33 M. P. Valley, W. Zhou, E. M. Hawkins, J. Shultz, J. J. Cali, T. Worzella, L. Bernad, T. Good, D. Good, T. L. Riss, D. H. Klaubert and K. V. Wood, Anal. Biochem., 2006, 359, 238-46.

34 A. Holt, D. F. Sharman, G. B. Baker and M. M. Palcic, Anal. Biochem., 1997, 244, 384-92.

35 K. Mccrickerd, N. Lensing and M. R. Yeomans, FOOD Qual. Prefer., 2015, 44, 130-138.

36 M. Halenár, M. Medved’ová, N. Maruniaková and A. Kolesárová, J. Microbiol., Biotechnol. Food Sci., 2013, 2, 1414-1423.

37 B. Qin and R. A. Anderson, Br. J. Nutr., 2012, 108, 581-587.

38 S. Frejnagel and M. Wroblewska, Ann. Nutr. Metab., 2010, 56, 163-9.

39 N. Kardum, M. Takić, K. Šavikin, M. Zec, G. Zdunić, S. Spasić and A. Konić-Ristić, J. Funct. Foods, 2014, 9, 89-97.

40 M. J. Millan, Prog. Neurobiol., 2003, 70, 83-244.

41 M. J. Millan, Pharmacol. Ther., 2006, 110, 135-370.

42 J. P. E. Spencer, D. Vauzour and C. Rendeiro, Arch. Biochem. Biophys., 2009, 492, 1-9.

43 C. Rendeiro, J. D. T. Gurreiro, C. M. Williams and J. P. E. Spencer, Proc. Nutr. Soc., 2012, 71, 246-262.

44 M. B. H. Youdim, D. Edmondson and K. F. Tipton, Nat. Rev. Neurosci., 2006, 7, 295-309.

45 A. K. Jäger and L. Saaby, Molecules, 2011, 16, 14711485.

46 H. X. Han, S. S. Hong, S. J. Hwang, K. M. Lee, Y. B. Hwang and S. J. Ro, Arch. Pharmacal Res., 2007, 30, 13-17.

47 J. Hellström, P. Mattila and R. Karjalainen, J. Food Compos. Anal., 2013, 31, 12-19.

48 K. Wilkes, L. R. Howard, C. Brownmiller and R. L. Prior, J. Agric. Food Chem., 2014, 62, 4018-4025.

49 M.-J. Bermudez-Soto, F.-A. Tomas-Barberan and M.-T. Garcia-Conesa, Food Chem., 2007, 102, 865-874.

50 J. M. Carbonell-Capella, M. Buniowska, F. J. Barba, M. J. Esteve and A. Frígola, Compr. Rev. Food Sci. Food Saf., 2014, 13, 155-171.

51 C. Morand, C. Manach, V. Crespy and C. Remesy, BioFactors, 2000, 12, 169-174.

52 K. Murota and J. Terao, Arch. Biochem. Biophys., 2003, 417, 12-7.

53 S. Schaffer and B. Halliwell, Genes Nutr., 2011, 7, 99-109.

54 I. Orhan, M. Kartal, F. Tosun and B. Sener, Z. Naturforsch., 2007, 62, 829-832. 